\title{
Orthonormal systems in spaces of number theoretical functions
}

\author{
Karl-Heinz Indlekofer ${ }^{\mathrm{a}, 1}$, Erdener Kaya ${ }^{\mathrm{b}}$, and Robert Wagner ${ }^{\mathrm{a}}$ \\ ${ }^{a}$ Faculty of Computer Science, Electrical Engineering and Mathematics, University of Paderborn, \\ D-33098 Paderborn, Germany \\ ${ }^{\mathrm{b}}$ Maritime Faculty Department of Basic Sciences, Mersin University, TR-33290 Mersin, Turkey \\ (e-mail: k-heinz@math.uni-paderborn.de; kayaerdener@mersin.edu.tr; robert.wagner43@gmx.de)
}

Zum Gedenken an Professor Jonas Kubilius anlässlich seines 100. Geburtstages

Received February 24, 2020; revised July 22, 2020

\begin{abstract}
In this paper, we consider some examples of set algebras $\mathcal{A}$ on $\mathbb{N}$. If $\mathcal{E}(\mathcal{A})$ is the set of simple functions on $\mathcal{A}$, then $\mathcal{L}^{* \alpha}(\mathcal{A})$ denotes the $\|\cdot\|_{\alpha}$-closure of $\mathcal{E}(\mathcal{A})$. Our aim is to determine a complete orthonormal system for the Hilbert space $L^{* 2}(\mathcal{A})$ in each regarded case. Here $L^{* 2}(\mathcal{A})$ denotes the quotient space $\mathcal{L}^{* 2}(\mathcal{A})$ modulo null-functions.

MSC: $11 \mathrm{~K} 65,11 \mathrm{~N} 37,11 \mathrm{~N} 64$

Keywords: integration on $\mathbb{N}$, almost even functions, almost periodic functions, limit periodic functions, almost multiplicative functions, almost $q$-multiplicative functions
\end{abstract}

\section{Introduction}

For a function $f: \mathbb{N} \rightarrow \mathbb{C}$, we define $\|\cdot\|_{\alpha}$ by

$$
\|f\|_{\alpha}:=\left\{\limsup _{x \rightarrow \infty} \frac{1}{x} \sum_{n \leqslant x}|f(n)|^{\alpha}\right\}^{1 / \alpha}, \quad 1 \leqslant \alpha<\infty .
$$

Let $\mathcal{L}^{\alpha}:=\left\{f: \mathbb{N} \rightarrow \mathbb{C}:\|f\|_{\alpha}<\infty\right\}$ be the linear space of functions on $\mathbb{N}$ with bounded seminorm $\|f\|_{\alpha}$. By $L^{\alpha}$ we denote the quotient space $\mathcal{L}^{\alpha}$ modulo null-functions (i.e., functions $f$ with $\|f\|_{\alpha}=0$ ). For $\alpha \geqslant 1$, the norm space $L^{\alpha}$ is complete [7].

Let $\mathcal{A}$ be an algebra of subsets of $\mathbb{N}$. Then

$$
\mathcal{E}(\mathcal{A}):=\left\{s \in \mathcal{E}: s=\sum_{j=1}^{m} \alpha_{j} \mathbf{1}_{A_{j}}, \alpha_{j} \in \mathbb{C}, A_{j} \in \mathcal{A}, j=1, \ldots, m, m \in \mathbb{N}\right\}
$$

denotes the space of simple functions on $\mathcal{A}$.

\footnotetext{
${ }^{1}$ Supported by a grant of Deutsche Forschungsgemeinschaft Project No. 289386657.
} 
Definition 1. For a given algebra $\mathcal{A}$ and $1 \leqslant \alpha<\infty$, the space $\mathcal{L}^{* \alpha}(\mathcal{A})$ is defined as the $\|\cdot\|_{\alpha}$-closure of $\mathcal{E}(\mathcal{A})$. A function $f \in \mathcal{L}^{* \alpha}(\mathcal{A})$ is called uniformly $(\mathcal{A})-\alpha$ summable. By $L^{\alpha}(\mathcal{A})$ we denote the quotient space $\mathcal{L}^{* \alpha}(\mathcal{A})$ modulo null functions.

Remark 1. If $\mathcal{A}=\mathcal{P}(\mathbb{N})$ is the algebra of all subsets of $\mathbb{N}$, then $\mathcal{L}^{* 1}(\mathcal{A})$ is the $\|\cdot\|_{1}$-closure of $l^{\infty}$ is the space $\mathcal{L}^{*}$ of uniformly summable functions introduced by Indlekofer [2].

Here we consider algebras $\mathcal{A}$ where every $A \in \mathcal{A}$ possesses an asymptotic density $\delta(A)$ defined by

$$
\delta(A):=\lim _{n \rightarrow \infty} \frac{1}{n} \sum_{\substack{m \leqslant n \\ m \in A}} 1
$$

if the limit exists. Then $\delta$ is finitely additive on $\mathcal{A}$, that is, $\delta$ is a content on $\mathcal{A}$.

We say that an arithmetical function $f$ possesses an (arithmetical) mean value $M(f)$ if

$$
M(f):=\lim _{n \rightarrow \infty} \frac{1}{n} \sum_{m \leqslant n} f(m)
$$

exists. If every $A \in \mathcal{A}$ possesses an asymptotic density, then every $f \in \mathcal{L}^{* 1}(\mathcal{A})$ possesses a mean value. Further, we define an inner product on $L^{* 2}(\mathcal{A})$ by

$$
\langle f, g\rangle:=M(f \bar{g}), \quad f, g \in L^{* 2}(\mathcal{A}) .
$$

This product is well-defined. For this, let $f, g \in L^{* 2}(\mathcal{A})$. If $\varepsilon>0$, then there exist $s_{1}, s_{2} \in \mathcal{E}(\mathcal{A})$ such that $\left\|f-s_{1}\right\|_{2}<\varepsilon$ and $\left\|g-s_{2}\right\|_{2}<\varepsilon$. Put $\varepsilon:=\varepsilon^{*} /\left(\|f\|_{2}+2\|g\|_{2}\right)$. Then

$$
\begin{aligned}
\left\|f \bar{g}-s_{1} \overline{s_{2}}\right\|_{1} & \leqslant\left\|f\left(\bar{g}-\overline{s_{2}}\right)\right\|_{1}+\left\|\left(f-s_{1}\right) \overline{s_{2}}\right\|_{1} \\
& \leqslant\|f\|_{2}\left\|\bar{g}-\overline{s_{2}}\right\|_{2}+\left\|f-\overline{s_{1}}\right\|_{2}\left\|\overline{s_{2}}\right\|_{2} \\
& \leqslant \varepsilon\left(\|f\|_{2}+2\|g\|_{2}\right) \leqslant \varepsilon^{*}
\end{aligned}
$$

and $f \bar{g} \in L^{* 1}(\mathcal{A})$. Since $L^{* 2}(\mathcal{A})$ is complete, the space $L^{* 2}(\mathcal{A})$ is a Banach space. Therefore the space $L^{* 2}(\mathcal{A})$ is a Hilbert space with the inner product defined above.

In this paper, we investigate examples of Hilbert spaces $L^{* 2}(\mathcal{A})$ together with associated (complete) orthonormal systems.

Remark 2. The described construction of $\mathcal{L}^{* \alpha}(\mathcal{A})$ was the starting point of an integration theory by Indlekofer (see $[4,5])$.

Embedding $\mathbb{N}$, endowed with the discrete topology, in the compact space $\beta \mathbb{N}$, the Stone-Čech compactification of $\mathbb{N}$, we get:

$$
\overline{\mathcal{A}}:=\{\bar{A}: A \in \mathcal{A}\}, \quad \text { where } \bar{A}:=\cos _{\beta \mathbb{N}} A,
$$

is an algebra in $\beta \mathbb{N}$ (for details, see $[4,5]$ ).

Let $\delta$ be a content on $\mathcal{A}$, that is, $\delta: \mathcal{A} \rightarrow \mathbb{R}_{\geqslant 0}$ is finitely additive, and define $\bar{\delta}$ on $\overline{\mathcal{A}}$ by

$$
\bar{\delta}(\bar{A})=\delta(A), \quad \bar{A} \in \overline{\mathcal{A}} .
$$

Then $\bar{\delta}$ is a pseudo-measure on $\overline{\mathcal{A}}$ and can be extended to a measure on $\sigma(\overline{\mathcal{A}})$, which we also denote by $\bar{\delta}$. This leads to the measure space $(\beta \mathbb{N}, \sigma(\overline{\mathcal{A}}), \bar{\delta})$. 


\section{Some Hilbert spaces and corresponding orthonormal systems}

\subsection{A simple case}

Let $\mathcal{A}_{0}$ be the algebra generated by the sets $A_{p}:=\{n \in \mathbb{N}: p \mid n\}, p$ prime, and put

$$
\delta\left(A_{p}\right):=M\left(\mathbf{1}_{A_{p}}\right)=\lim _{n \rightarrow \infty} \frac{1}{n} \sum_{\substack{m \leqslant n \\ p \mid m}} 1=\frac{1}{p} .
$$

Note that the following relations of the characteristic functions

$$
\mathbf{1}_{A \cap B}=\mathbf{1}_{A} \cdot \mathbf{1}_{B}, \quad \mathbf{1}_{A \backslash B}=\mathbf{1}_{A}-\mathbf{1}_{A} \cdot \mathbf{1}_{B}, \quad \mathbf{1}_{A \cup B}=\mathbf{1}_{A}+\mathbf{1}_{B}-\mathbf{1}_{A} \cdot \mathbf{1}_{B}
$$

imply that the characteristic function of a set $A \in \mathcal{A}$ is a finite linear combination of products $\mathbf{1}_{A_{p_{1}}} \cdots \mathbf{1}_{A_{p_{r}}}$. Thus the asymptotic density $\delta(A)$ exists for all $A \in \mathcal{A}_{0}$.

For every prime $p$, put

$$
h_{p}:=p \mathbf{1}_{A_{p}}-1
$$

and define $h_{n}: \mathbb{N} \rightarrow \mathbb{Z}$ by $h_{n}=1$ for $n=1$ and

$$
h_{n}:=\prod_{p \mid n} h_{p} \quad \text { for every square-free } n \in \mathbb{N}
$$

Obviously, for every prime $p$,

$$
M\left(h_{p}\right)=0, \quad M\left(h_{p}^{2}\right)=p-1 .
$$

Now, if $f: \mathbb{N} \rightarrow \mathbb{C}$ is such that $M(f)$ exists and $f(p m)=f(m)$ for all $m \in \mathbb{N}$, then we conclude that

$$
\sum_{m \leqslant x} h_{p}(m) f(m)=p \sum_{p m \leqslant x} f(p m)-\sum_{m \leqslant x} f(m)=p \sum_{m \leqslant x / p} f(m)-\sum_{m \leqslant x} f(m)
$$

and $M\left(h_{p} f\right)=0$, that is,

$$
M\left(h_{n}\right)=0 \quad \text { if } \mu^{2}(n)=1, n>1,
$$

and

$$
M\left(h_{n} h_{n^{\prime}}\right)=0 \quad \text { if } \mu^{2}(n)=\mu^{2}\left(n^{\prime}\right)=1 \text { and } n \neq n^{\prime} .
$$

In the same way, we obtain

$$
M\left(h_{p}^{2} f\right)=(p-1) M(f)
$$

By induction this leads to

$$
M\left(h_{n}^{2}\right)=\varphi(n) \quad \text { if } \mu^{2}(n)=1 .
$$

Putting $h_{n}^{*}:=(\varphi(n))^{-1 / 2} h_{n}\left(\mu^{2}(n)=1\right)$, we have shown the following:

Theorem 1. The set $\left\{h_{n}^{*}: n\right.$ square-free $\}$ is a complete orthonormal system for $L^{* 2}\left(\mathcal{A}_{0}\right)$.

Remark 3. We easily to see that the function $h_{n}: \mathbb{N} \rightarrow \mathbb{Z}$ satisfies $h_{n_{1} n_{2}}=h_{n_{1}} \cdot h_{n_{2}}$ if $\left(n_{1}, n_{2}\right)=1$ and $\mu\left(n_{1}\right)=\mu\left(n_{2}\right)=1$, that is, $n=1$, or $n$ is a product of an even number of different primes.

Remark 4. Every $f \in \mathcal{E}\left(\mathcal{A}_{0}\right)$ can be written as a linear combination of multiplicative $g_{j}$ such that $g_{j}\left(p^{l}\right)=1$ for all $p \geqslant k_{j}$ and $l \in \mathbb{N}$, since $g=1-\mathbf{1}_{A_{p}}$ is multiplicative. 


\subsection{Almost even functions}

For primes $p$ and $k=0,1,2, \ldots$, let $A_{p^{k}}:=\left\{n \in \mathbb{N}: p^{k} \mid n\right\}$ be the set of natural numbers divisible by $p^{k}$. Let $\mathcal{A}_{1}$ be the algebra generated by the sets $\left\{A_{p^{k}}\right\}$. Then, for all $A_{p^{k}}$, the asymptotic density $\delta\left(A_{p^{k}}\right)$ exists and equals $1 / p^{k}$, and, as before, the asymptotic density $\delta(A)$ exists for all $A \in \mathcal{A}_{1}$.

Schwarz and Spilker [8, Chap. VI] considered the space $\mathcal{B}$ of even functions and characterized the sets of $\alpha$-almost even functions (see also [3]).

It is well known that $\mathcal{E}\left(\mathcal{A}_{1}\right)$ equals $\mathcal{B}$ and $\mathcal{L}^{\alpha}\left(\mathcal{A}_{1}\right)$ is exactly the space of $\alpha$-almost even functions (see [5]).

Remark 5. Every $f \in \mathcal{E}\left(\mathcal{A}_{1}\right)$ can be written as a linear combination of multiplicative functions $g_{j}$ such that $g_{j}\left(p^{l}\right)=1$ for all $p \geqslant k_{j}$ and $l \in \mathbb{N}$.

Remark 6. Let $f: \mathbb{N} \rightarrow \mathbb{C}$ be a multiplicative function such that $|f| \leqslant 1$. Then the following statements hold.

(i) If $M(f) \neq 0$, then $f \in \mathcal{L}^{* \alpha}\left(\mathcal{A}_{1}\right)$ for every $\alpha \geqslant 1$.

(ii) $M(|f|)=0$ if and only if $\sum_{p \text { prime }}(1-|f(p)|) / p=\infty$; especially, if $\sum_{p \text { prime, } f(p)=0} 1 / p=\infty$, then $M(|f|)=0$.

(iii) $M(|f|)=0$ if and only if $M\left(|f|^{2}\right)=0$.

Put

$$
h_{p}:=p \mathbf{1}_{A_{p}}-1 \text { for prime } p
$$

and

$$
h_{p^{k}}:=p^{k} \mathbf{1}_{A_{p^{k}}}-p^{k-1} \mathbf{1}_{A_{p^{k-1}}} \quad \text { for } k>2 .
$$

Define $h_{n}=1$ for $n=1$ and

$$
h_{n}:=\prod_{p^{k} \| n} h_{p^{k}} \quad \text { for } n>1
$$

Putting

$$
h_{n}^{*}=\frac{1}{(\varphi(n))^{1 / 2}} h_{n},
$$

where $\varphi$ is Euler's function, it is easy to show (see above) that $\left\{h_{n}^{*}\right\}$ is an orthonormal system. We conclude by the following:

Theorem 2. The set $\left\{h_{n}^{*}: n \in \mathbb{N}\right\}$ is a complete orthonormal system for $L^{* 2}\left(\mathcal{A}_{1}\right)$.

Remark 7. The functions $h_{n}$ appear in a very natural way. It is not difficult to show (see [8, pp. 16-17]) that $h_{n}$ is just the Ramanujan sum $c_{n}$ for every $n$.

\subsection{Limit periodic functions}

Let $\mathcal{A}_{2}$ be the algebra generated by all residue classes $A_{a, r}:=\{n \in \mathbb{N}: n \equiv a \bmod r\}, 1 \leqslant a \leqslant r, r \in \mathbb{N}$.

Here again the asymptotic density $\delta$ is a finite additive function on $\mathcal{A}_{2}$. Then we have the following lemma.

Lemma 1. $\mathcal{E}\left(\mathcal{A}_{2}\right)$ is the space of all periodic functions on $\mathbb{N}$.

The space $L^{* \alpha}\left(\mathcal{A}_{2}\right)$ is the space of $\alpha$-limit-periodic functions.

Defining $e_{a / r}: \mathbb{N} \rightarrow \mathbb{C}$ by

$$
e_{a / r}(n):=\exp \left(2 \pi i \frac{a}{r} n\right)
$$

we have the following result (see [8, p. 207]).

Theorem 3. The set $\left\{e_{a / r}: 1 \leqslant a \leqslant r, \operatorname{gcd}(a, r)=1, r=1,2, \ldots\right\}$ is a complete orthonormal system in $L^{* 2}\left(\mathcal{A}_{2}\right)$. 


\subsection{Almost periodic functions}

For $\beta \in \mathbb{R}$, the function $e_{\beta}: \mathbb{N} \rightarrow \mathbb{C}$ defined by

$$
e_{\beta}(n)=\exp (2 \pi \mathrm{i} \beta n), \quad n \in \mathbb{N},
$$

possesses a mean value $M\left(e_{\beta}\right)$.

Let $\mathcal{C}$ be the family of all half-open subsets of $[0,1]$ and denote by $\mathcal{A}_{3}$ the algebra generated by the sets $A(\beta, E):=\{n \in \mathbb{N}:\{\beta n\} \in E\}$, where $\beta \in[0,1), E \in \mathcal{C}$, and $\beta n=[\beta n]+\{\beta n\}(0 \leqslant \beta n<1)$. Then (see [8, p. 207]) we have the following:

Theorem 4. The set $\left\{e_{\beta}: \beta \in[0,1]\right\}$ is a complete orthonormal system in $\mathcal{L}^{* 2}\left(\mathcal{A}_{3}\right)$.

\subsection{Almost multiplicative functions}

Let $f$ be a multiplicative function taking only the values $\{-1,0,1\}$ and define the sets

$$
A_{f}^{+}:=\{n: f(n)=1\}, \quad A_{f}^{0}:=\{n: f(n)=0\}, \quad \text { and } \quad A_{f}^{-}:=\{n: f(n)=-1\}
$$

with characteristic functions $f^{+}, f^{0}$, and $f^{-}$, respectively. Obviously,

$$
f^{+}=\frac{1}{2}(|f|+f), \quad f^{0}=1-f^{+}-f^{-}, \quad f^{-}=\frac{1}{2}(|f|-f) .
$$

We define the algebra $\mathcal{A}_{4}$ to be the algebra generated by the sets $A_{f}^{+}, A_{f}^{0}, A_{f}^{-}$for all multiplicative functions $f$ with $f(\mathbb{N}) \subset\{-1,0,1\}$. Every $A \in \mathcal{A}_{4}$ possesses an asymptotic density by Wirsing's theorem. An arbitrary element $A$ of $\mathcal{A}_{4}$ has a characteristic function that is a linear combination of such multiplicative functions. Thus the asymptotic density $\delta(A)$ exists. Let $\mathcal{E}\left(\mathcal{A}_{4}\right)$ be the vector space of simple functions on $\mathcal{A}_{4}$. Let $\mathcal{L}^{* \alpha}\left(\mathcal{A}_{4}\right)$ be the $\|\cdot\|_{\alpha}$-closure of $\mathcal{E}\left(\mathcal{A}_{4}\right)$.

DEFINITION 2. A function $f \in \mathcal{L}^{* \alpha}\left(\mathcal{A}_{4}\right)$ is called an $\alpha$-almost multiplicative function.

First, we show $\mathcal{A}_{1} \subset \mathcal{A}_{4}$. For the proof, consider

$$
f^{*}(n):=\left(1-\mathbf{1}_{A_{p^{k}}}\right)(n)= \begin{cases}0, & p^{k} \mid n \\ 1 & \text { otherwise }\end{cases}
$$

Then $f^{*}$ is multiplicative. Since $\mathbf{1}_{\mathbb{N} \backslash A_{p^{k}}}=\mathbf{1}_{\mathbb{N}}-\mathbf{1}_{A_{p^{k}}}=1-\mathbf{1}_{A_{p^{k}}} \in \mathcal{E}\left(\mathcal{A}_{4}\right)$, we have $\mathbb{N} \backslash A_{p^{k}} \in \mathcal{A}_{4}$. This implies that $A_{p^{k}} \in \mathcal{A}_{4}$.

Since $h_{n} \in \mathcal{E}\left(\mathcal{A}_{1}\right)$, we have $h_{n} \in \mathcal{E}\left(\mathcal{A}_{4}\right)$. Every $h_{n}$ can be written as a finite linear combination of $\mathbf{1}_{A_{p_{1}^{\alpha}}} \cdots \cdot \mathbf{1}_{A_{p_{m}^{\alpha}}}$, where $m \in \mathbb{N}$.

Theorem 5. Let $f: \mathbb{N} \rightarrow \mathbb{R}$ be multiplicative with $|f| \leqslant 1$. Then $f \in \mathcal{L}^{* \alpha}\left(\mathcal{A}_{4}\right)$ for all $\alpha \geqslant 1$.

Proof. Put $f=|f| \operatorname{sign}_{f}$, where $\operatorname{sign}_{f}$ is multiplicative with

$$
\left(\operatorname{sign}_{f}\right)\left(p^{l}\right)= \begin{cases}1 & \text { if } f\left(p^{l}\right)>0 \\ 0 & \text { if } f\left(p^{l}\right)=0 \\ -1 & \text { if } f\left(p^{l}\right)<0\end{cases}
$$


Since $|f| \in \mathcal{L}^{* \alpha}\left(\mathcal{A}_{1}\right)$ and $\operatorname{sign} f \in \mathcal{E}\left(\mathcal{A}_{4}\right)$, we find $s_{1}, s_{s} \in \mathcal{E}\left(\mathcal{A}_{4}\right)$ such that $\left\|f\left|-s_{1}\right|\right\|_{\alpha}^{\alpha} \leqslant \varepsilon^{\alpha}$ and $\left\|\operatorname{sign} f-s_{2}\right\|_{\alpha}^{\alpha} \leqslant \varepsilon^{\alpha}$. Note that there exist $c_{1}(\alpha), c_{2}(\alpha)>0$ such that

$$
\left\|s_{2}\right\|_{\alpha}^{\alpha} \leqslant\left\|1+\left(s_{2}-|f|\right)\right\|_{\alpha}^{\alpha} \leqslant c_{1}(\alpha)
$$

and

$$
(|a|+|b|)^{\alpha} \leqslant c_{2}(\alpha)\left(|a|^{\alpha}+|b|^{\alpha}\right)
$$

Put $\varepsilon^{\alpha}:=\varepsilon^{* \alpha} /\left(1+c_{1}(\alpha) c_{2}(\alpha)\right)$. Then

$$
\begin{aligned}
\left\|f-s_{1} s_{2}\right\|_{\alpha}^{\alpha} & =c_{2}(\alpha)\left\{\left\||f|\left(\operatorname{sign}_{f}-s_{2}\right)\right\|_{\alpha}^{\alpha}+\left\|\left(|f|-s_{1}\right) s_{2}\right\|_{\alpha}^{\alpha}\right\} \\
& \leqslant c_{2}(\alpha)\left\{\left\|\left(\operatorname{sign}_{f}-s_{2}\right)\right\|_{\alpha}^{\alpha}+\left\|\left(|f|-s_{1}\right)\right\|_{\alpha}^{\alpha}\left\|s_{2}\right\|_{\alpha}^{\alpha}\right\} \\
& \leqslant c_{2}(\alpha) \varepsilon^{\alpha}+\varepsilon^{\alpha} c_{2}(\alpha) c_{1}(\alpha)<\varepsilon^{* q} .
\end{aligned}
$$

This proves Theorem 5 .

Next, we construct an orthonormal system for the space $L^{* 2}\left(\mathcal{A}_{4}\right)$.

Let $\mathcal{R}_{0}$ be the set of all multiplicative functions with $f(\mathbb{N}) \subset\{-1,0,1\}$ and $M(|f|) \neq 0$. Define the relation $\sim$ on $\mathcal{R}_{0}$ by

$$
f \sim g \text { if and only if } \sum_{\substack{p \\ f(p) \neq g(p)}} \frac{1}{p}<\infty .
$$

Observe, that in this case, by (ii) of Remark $6, \sum_{p, f(p)=0} 1 / p<\infty$. Obviously, $\sim$ is an equivalence relation on $\mathcal{R}_{0}$.

Now choose a representative from each residue class that takes only the values \pm 1 and denote this set by $\mathcal{F}_{1}$. Then $\mathcal{F}_{1}$ forms an orthonormal system. For this, let $f, g \in \mathcal{F}_{1}$ and observe that $\sum_{p, f(p) \neq g(p)} 1 / p=\infty$. Then, by (ii) of Remark 6, $M(f \bar{g})=\langle f, g\rangle=0$. Furthermore, for $f \in \mathcal{F}_{1}$, we have $f^{2}=1$ and $\langle f, f\rangle=M\left(f^{2}\right)=1$.

This shows that $\mathcal{F}_{1}$ is an orthonormal system in $L^{* 2}\left(\mathcal{A}_{4}\right)$. Consider, for $f \in \mathcal{F}_{1}$, the system

$$
\mathcal{F}_{2}:=\left\{f h_{n}^{*}: f \in \mathcal{F}_{1}, n \in \mathbb{N}\right\},
$$

where $h_{n}^{*}$ is the normalized function (2.1).

Theorem 6. $\mathcal{F}_{2}$ is a complete orthonormal system for $L^{* 2}\left(\mathcal{A}_{4}\right)$.

Proof. First, we show that $\mathcal{F}_{2}$ is an orthonormal system. For this, let $h_{n}^{*} f \neq h_{\tilde{n}}^{*} g$. This holds if and only if $f \neq g$ and $n, \tilde{n}$ are arbitrary or $f=g$ and $n \neq \tilde{n}$. Assume that $f \neq g$ and $n, \tilde{n}$ are arbitrary. Then

$$
\left\langle h_{n}^{*} f, h_{\bar{n}}^{*} g\right\rangle=M\left(h_{n}^{*} f h_{\bar{n}} g\right)=M\left(f g h^{*}\right),
$$

where $h^{*}$ is (see Remark 6) a finite linear combination of multiplicative functions $g_{j}$ with $\left|g_{j}\right|=1$ and $g_{j}(p)=1$ for $p>k_{j}$. Therefore

$$
\sum_{p} \frac{1-f(p) g(p) g_{j}(p)}{p}=\infty \quad \text { and } \quad M\left(f g g_{j}\right)=0 .
$$

So we obtain $\left\langle h_{n}^{*} f, h_{\bar{n}}^{*} g\right\rangle=0$ if $f \neq g$. In the case $f=g$ and $n \neq \bar{n}$, obviously, $\left\langle h_{n}^{*} f, h_{\bar{n}}^{*} f\right\rangle=M\left(h_{n}^{*} h_{\bar{n}}^{*}\right)=0$. Since $\left\langle h_{n}^{*} f, h_{\bar{n}}^{*} f\right\rangle=M\left(\left|h_{n}^{*} f\right|^{2}\right)=1, \mathcal{F}_{2}$ is an orthonormal system. 
For the proof of the completeness of $\mathcal{F}_{2}$, let $g \in \mathcal{L}^{*}\left(\mathcal{A}_{4}\right)$. Then $g$ can be approximated in the $\|\cdot\|_{2}$ norm by some $g^{*} \in \mathcal{E}\left(\mathcal{A}_{4}\right)$,

$$
g^{*}=\sum_{j=1}^{m} \alpha_{j} \mathbf{1}_{A_{j}}, \quad \alpha_{j} \in \mathbb{C}, A_{j} \in \mathcal{A}_{4} .
$$

Note that $\mathbf{1}_{A}$ for $A \in \mathcal{A}_{4}$ is a finite linear combination of products of multiplicative functions $f$ taking only the values $\{-1,0,1\}$.

Therefore it suffices to prove that each real-valued multiplicative function $f$ with values $f(\mathbb{N}) \subset\{-1,0,1\}$ can be approximated by a linear combination of functions from $\mathcal{F}_{2}$. Choose $g \in \mathcal{F}_{1}$ that is equivalent to $f$. Then $f=h g$ where $h=f g$, since $g^{2}=1$. Then

$$
\sum_{p} \frac{1-h(p)}{p}=\sum_{p} \frac{2}{p}<\infty
$$

and $h \in \mathcal{L}^{* 2}\left(\mathcal{A}_{1}\right)$. Thus $h$ can be approximated by a linear combination of functions $h_{1}, \ldots, h_{m}$, that is, for $\varepsilon>0$, there exist $\alpha_{j} \in \mathbb{C}$ such that

$$
\left\|h-\sum_{j=1}^{m} \alpha_{j} h_{j}\right\|_{2}<\varepsilon, \quad \text { which implies }\left\|f-\sum_{j=1}^{m} \alpha_{j} h_{j} g\right\|_{2}<\varepsilon .
$$

This ends the proof of the completeness of $\mathcal{F}_{2}$.

\section{6 $q$-ary almost even functions}

First, we introduce $q$-multiplicative functions. Let $q \geqslant 2$ be an integer, and let $\mathbb{A}=\{0,1, \ldots, q-1\}$. The $q$-ary expansion of some $n \in \mathbb{N}_{0}$ is defined as the unique sequence $\varepsilon_{0}(n), \varepsilon_{1}(n), \ldots$ for which

$$
n=\sum_{j=0}^{\infty} \varepsilon_{j}(n) q^{j}, \quad \varepsilon_{j}(n) \in \mathbb{A} .
$$

The numbers $\varepsilon_{0}(n), \varepsilon_{1}(n), \ldots$ are called the digits in the $q$-ary expansion of $n$. In fact, $\varepsilon_{r}(n)=0$ if $r>\log n / \log q$. A function $f: \mathbb{N}_{0} \rightarrow \mathbb{C}$ is called q-multiplicative if $f(0)=1$ and for every $n \in \mathbb{N}_{0}$,

$$
f(n)=\prod_{j=0}^{\infty} f\left(\varepsilon_{j}(n) q^{j}\right)
$$

Let the algebra $\mathcal{A}_{5}$ be generated by the sets $A_{j, a}:=\left\{n \in \mathbb{N}: \varepsilon_{j}(n)=a\right\}, j \in \mathbb{N}_{0}, a \in \mathbb{A}$. Every $A \in \mathcal{A}_{5}$ possesses an asymptotic density $\delta(A)$.

Let $\mathcal{L}^{* 1}\left(\mathcal{A}_{5}\right)$ be the $\|\cdot\|_{1}$-closure of $\mathcal{E}\left(\mathcal{A}_{5}\right)$. Here $\mathcal{E}\left(\mathcal{A}_{5}\right)$ is called the space of $q$-ary even functions. Then $\mathcal{L}^{* 1}\left(\mathcal{A}_{5}\right)$ is called the space of $q$-ary almost even functions.

Remark 8 . Let $f$ be a real-valued $q$-multiplicative function of modulus $\leqslant 1$. Then the mean values $M(|f|)$ and $M(f)$ always exist (see [6]). Especially, we have:

(i) If $\|f\|_{1}=M(|f|)>0$, then

$$
\sum_{j=0}^{\infty} \sum_{a \in \mathbb{A}}\left(1-\left|f\left(a q^{j}\right)\right|\right)<\infty
$$


(ii) If

$$
\sum_{a \in \mathbb{A}} f\left(a q^{j}\right) \neq 0 \quad \text { for all } j \in \mathbb{N}_{0} \text { and } \sum_{j=0}^{\infty} \sum_{a \in \mathbb{A}}\left(1-f\left(a q^{j}\right)\right)<\infty
$$

then $M(f) \neq 0$.

As an immediate consequence, we have the following:

Corollary 1. Let $f$ be a real-valued q-multiplicative function of modulus $\leqslant 1$. If

$$
\sum_{j=0}^{\infty} \sum_{a \in \mathbb{A}}\left(1-f\left(a q^{j}\right)\right)<\infty
$$

then $f \in \mathcal{L}^{* 1}\left(\mathcal{A}_{5}\right)$.

This ends Remark 8.

Let $\mathcal{L}^{* 2}\left(\mathcal{A}_{5}\right)$ be the $\|\cdot\|_{2}$-closure of $\mathcal{E}\left(\mathcal{A}_{5}\right)$. Then we define a complete orthonormal system for the space $L^{* 2}\left(\mathcal{A}_{5}\right)$.

Theorem 7. The set $\left\{h_{a_{0}, \ldots, a_{r}}\right\}$ of $q$ - multiplicative functions with

$$
h_{a_{0}, \ldots, a_{r}}(n):=\prod_{j=0}^{r} \exp \left(\frac{2 \pi \mathrm{i} a_{j}}{q} \varepsilon_{j}(n)\right),
$$

$a_{j} \in \mathbb{A}, j=0, \ldots, r, r \in \mathbb{N}_{0}$, is a complete orthonormal system for $L^{* 2}\left(\mathcal{A}_{5}\right)$.

The proof is easy and is left to the reader.

\subsection{Almost $q$-multiplicative functions}

Let $f$ be a $q$-multiplicative function taking only the values $\{-1,0,1\}$ and define the sets

$$
A_{f}^{+}:=\{n: f(n)=1\}, \quad A_{f}^{0}:=\{n: f(n)=0\}, \quad \text { and } \quad A_{f}^{-}:=\{n: f(n)=-1\}
$$

with characteristic functions $f^{+}, f^{0}$, and $f^{-}$, respectively. We denote by $\mathcal{A}_{6}$ the algebra generated by the sets $A_{f}^{+}, A_{f}^{0}, A_{f}^{-}$for all $q$-multiplicative $f$ with $f(\mathbb{N}) \subset\{-1,0,1\}$.

An arbitrary element $A$ of $\mathcal{A}_{6}$ has a characteristic function that is a linear combination of $q$-multiplicative functions. From this and by the theorem of Delange [1] the asymptotic density $\delta(A)$ exists. Let $\mathcal{E}\left(\mathcal{A}_{6}\right)$ be the space of simple functions on $\mathcal{A}_{6}$. Let $\mathcal{L}^{*}\left(\mathcal{A}_{6}\right)$ be the $\|\cdot\|_{1}$-closure of $\mathcal{E}\left(\mathcal{A}_{6}\right)$.

DEFInITION 3. Functions $f \in \mathcal{L}^{*}\left(\mathcal{A}_{6}\right)$ are called almost q-ary multiplicative functions.

Next, we define a complete orthonormal system for $L^{* 2}\left(\mathcal{A}_{6}\right)$.

Let $\mathcal{G}:=\left\{f: \mathbb{N} \rightarrow \mathbb{R}: f q-\right.$ multiplicative, $f(n) \in\{-1,0,1\}$ for all $n \in \mathbb{N}$ with $\left.\|f\|_{2} \neq 0\right\}$. Define the relation $\sim$ on $\mathcal{G}$ by

$$
f \sim g \quad \text { if and only if } \sum_{l=0}^{\infty} \sum_{a=0}^{q-1}\left(1-f\left(a q^{l}\right) g\left(a q^{l}\right)\right)<\infty .
$$

Obviously, $\sim$ is an equivalence relation on $\mathcal{G}$. 
Now from each equivalence class we choose a representative that is $\neq 0$ for all $n \in \mathbb{N}$. We denote this set of representatives by $\mathcal{F}_{3}$. We consider $\mathcal{F}_{4}:=\left\{h_{a_{0}, \ldots, a_{r}} f: f \in \mathcal{F}_{3}, a_{j} \in A, j=0, \ldots, r, r \in \mathbb{N}\right\}$ and show the following:

Theorem 8. $\mathcal{F}_{4}$ is a complete orthonormal system for $L^{* 2}\left(\mathcal{A}_{6}\right)$.

Proof. First, we show that $\mathcal{F}_{4}$ is an orthonormal system. In the case $g_{1}=g_{2}=g$, since $g^{2}=1$, we have

$$
M\left(h_{a_{0}, \ldots, a_{r}} g \bar{h}_{b_{0}, \ldots, b_{s}} g\right)=M\left(h_{a_{0}, \ldots, a_{r}} \bar{h}_{b_{0}, \ldots, b_{r}}\right)=0
$$

if $h_{a_{0}, \ldots, a_{r}} \neq h_{b_{0}, \ldots, b_{r}}$ and 1 otherwise. If $g_{1} \neq g_{2}$, then

$$
\left(h_{a_{0}, \ldots, a_{r}} g_{1} \bar{h}_{b_{1}, \ldots, b_{s}} g_{2}\right)\left(a q^{j}\right)=\left(g_{1} g_{2}\right)\left(a q^{j}\right)
$$

if $j$ is large enough. Obviously, $M\left(g_{1} g_{2}\right)=0$, and $\mathcal{F}_{4}$ is an orthonormal system.

To prove the completeness of $\mathcal{F}_{4}$, it suffices to show that every $q$-multiplicative $f$ with $M(|f|) \neq 0$ and $f(n) \in\{-1,0,1\}$ for all $n \in \mathbb{N}_{0}$ can be approximated by linear combinations of elements of $\mathcal{F}_{4}$.

Let $f$ be such a function. Then $f=|f| \operatorname{sign}_{f}$ and $\operatorname{sign}_{f} \sim g, g \in \mathcal{F}_{3}$, and

$$
f=|f| \operatorname{sign}_{f} g^{2}=\left(|f| \operatorname{sign}_{f} g\right) g .
$$

Now $|f| \operatorname{sign}_{f} g$ is a $q$-ary even function and can therefore be approximated by a linear combination of some $h_{a_{0}, \ldots, a_{r}}$. This proves Theorem 8 .

Open Access. This article is licensed under a Creative Commons Attribution 4.0 International License, which permits use, sharing, adaptation, distribution and reproduction in any medium or format, as long as you give appropriate credit to the original author(s) and the source, provide a link to the Creative Commons licence, and indicate if changes were made. The images or other third party material in this article are included in the article's Creative Commons licence, unless indicated otherwise in a credit line to the material. If material is not included in the article's Creative Commons licence and your intended use is not permitted by statutory regulation or exceeds the permitted use, you will need to obtain permission directly from the copyright holder. To view a copy of this licence, visit http://creativecommons.org/licenses/by/4 .0/

\section{References}

1. H. Delange, Sur les fonctions $q$-additives ou $q$-multiplicatives, Acta Arith., 21:285-298, 1972.

2. K.-H. Indlekofer, A mean-value theorem for multiplicative arithmetical functions, Math. Z., 172:255-271, 1980.

3. K.-H. Indlekofer, Some remarks on almost-even and almost-periodic functions, Arch. Math., 37:353-358, 1981.

4. K.-H. Indlekofer, A new method in probabilistic number theory, in J. Galambos and I. Kátai (Eds.), Probability Theory and Applications, Math. Appl., Springer, Vol. 80, Springer, Dordrecht, 1992, pp. 299-308.

5. K.-H. Indlekofer, New approach to probabilistic number theory - compactifications and integration, in S. Akiyama et al. (Eds.), Probability and Number Theory - Kanazawa 2005. Proceedings of the International Conference on Probability and Number Theory, Kanazawa, Japan, June 20-24, 2005, Mathematical Society of Japan, Tokyo, 2007, pp. 133-170.

6. K.-H. Indlekofer, Y.-W. Lee, and R. Wagner, Mean behaviour of uniformly summable $q$-multiplicative functions, Ann. Univ. Sci. Budap. Rolando Eötvös, Sect. Comput., 25:171-294, 2005.

7. J. Knopfmacher, Fourier analysis of arithmetical functions, Annali Mat. Pura Appl. (4), 109:177-201, 1976.

8. W. Schwarz and J. Spilker, Arithmetical Functions: An Introduction to Elementary and Analytic Properties of Arithmetic Functions and to Some of Their Almost-Periodic Properties, Cambridge Univ. Press, Cambridge, 1994. 